\title{
Evolutionary Dynamics of Large Numts in the Human Genome: Rarity of Independent Insertions and Abundance of Post-Insertion Duplications
}

\author{
Einat Hazkani-Covo, ${ }^{1}$ Rotem Sorek, ${ }^{1,2}$ Dan Graur ${ }^{1}$ \\ ${ }^{1}$ Department of Zoology, George S. Wise Faculty of Life Sciences, Tel Aviv University, Ramat Aviv 69978, Israel \\ ${ }^{2}$ Compugen Ltd., 72 Pinchas Rosen St., Tel Aviv 69512, Israel
}

Received: 12 July 2002 / Accepted: 9 September 2002

Abstract. We determined the phylogenetic positions of 82 large nuclear pseudogenes of mitochondrial origin (numts) within the human genome. For each numt, two possibilities pertaining to its origin were considered: (1) independent insertion from the mitochondria into the nucleus, or (2) genomic duplication subsequent to the insertion. A significant increase in the rate of numt accumulation is seen after the divergence of Platyrrhini (New World monkeys) from the Catarrhini (Old World monkeys, apes and humans). By using pairwise phylogenetic analyses, we were able to demonstrate that this peak in numt accumulation is mostly the result of duplication of preexisting nuclear numts rather than the result of an increase in mitochondrial-sequence insertion. In fact, only about a third of all the numt repertoire in the human nuclear genome is due to insertions of mitochondrial sequences, the rest originated as duplications of preexisting numts. Hence, we conclude that numt insertion occurs at a much lower rate than previously reported. As expected under the assumption that genomic duplications occur at rates that are uninfluenced by content, older numts were found to be duplicated more times than recently inserted ones.

Key words: Numts - Human genome - Promiscuous DNA - Gene duplicaton - Pseudogenes - Primates

\section{Introduction}

Starting with the findings of Stern and Lonsdale (1982) on the transfer of genetic information among genomes, hundreds of studies have documented the ubiquity of genetic-information flow between organelles and between organelles and the nucleus (e.g., Blanchard and Schmidt 1995; Collura and Stewart 1995; Fukuda et al. 1985; Lopez et al. 1994). This type of "disrespect" for genomic barriers has been dubbed "promiscuous DNA" (Ellis 1982; Lewin 1983). To date, examples have been found for five out of the six possible types of gene transfer among genomes: chloroplast to mitochondria, mitochondria to chloroplast, chloroplast to nucleus, nucleus to mitochondria, and mitochondria to nucleus (Thorsness and Weber 1996).

While the transfer of functional mitochondrial genes into the nucleus has most probably ceased before the emergence of animals, approximately 1,000 million years ago (Boore 1999), the integration of functionless mitochondrial sequences into the nuclear genome has continued unremittingly, and nuclear pseudogenes of mitochondrial origin or numts (pronounced "new-mights", Lopez et al. 1994) have been described in numerous eukaryotes (Bensasson et al. 2001). All mammalian numts studied to date were found to be functionless, and it is thought that because of the differences between the nuclear and mitochondrial genetic codes, they became pseudogenes immediately on arrival into the nucleus. Numts have an uneven taxonomic and chromosomal distribution, 
but so far no diagnostic features have been described for the regions flanking the numt integration sites (Bensasson et al. 2001). Gene transfer from the mitochondria to the nucleus most probably occurs through direct DNA transfer, rather than through cDNA-mediated transfer (Henze and Martin 2001).

Recently, the full numt repertoire of the human nuclear genome was published (Mourier et al. 2001; Woischnik and Moraes 2002). On the basis of phylogenetic analyses, both groups concluded that the evolutionary process of numt insertion is continuous (Bensasson et al. 2001), and that it occurs at fairly rapid rates. However, we find their phylogenetic analyses incomplete, especially since they regard all numts as independent mitochondrial insertions and neglect the possibility of post-insertion nuclear duplication (e.g., Bensasson et al. 2000). In this study, we attempted to reconstruct the evolutionary dynamics of numt accumulation. In particular, we determined for each numt whether it was derived independently through the integration of a mitochondrial sequence or whether it was created through the nuclear-genome duplication of a preexisting numt.

\section{Materials and Methods}

Data Collection. The FASTA algorithm (Pearson and Lipman 1988) was used to search each of the human chromosomes (ftp:// ncbi.nlm.nih.gov/genomes/H_sapiens/) for regions of similarity with the human mitochondrial sequence (Genebank, NC_001807). Ninety-four hits that were longer than 1,000 bp were selected for further analysis. After filtering overlapping results and choosing the ones that had the longer hits, we used the Smith-Waterman algorithm (Smith and Waterman 1981) to join closely spaced $(<100 \mathrm{~Kb})$ hits that were found on the same contig and in the same orientation. The algorithm was employed to ensure that each numt in our analysis appears only once, i.e., that it was not artificially divided into segments. These procedures reduced the number of hits to 82 numts.

Phylogenetic Analysis of numts. Ten full mitochondrial sequences were selected for phylogenetic analysis and were aligned using ClustalW (Higgins et al. 1996). A user tree (Fig. 1A) was built for fully sequenced mitochondrial genomes from eight primates, a sister taxon (Tupaia belangeri, Scandentia), and an outgroup (Phoca vitulina, Pinnipedia, Carnivora). The taxa were chosen on the basis of complete-mitochondrial sequence availability and the possibility of building a taxonomically undisputed phylogenetic tree (Goodman et al. 1998). Genebank accession numbers for the mitochondrial sequences are: NC_001807 for human (Homo sapiens), NC_001643 for chimpanzee (Pan troglodytes), NC_001645 for gorilla (Gorilla gorilla), NC_001646 for orangutan (Pongo pygmaeus), NC_002082 for white-handed gibbon (Hylobates lar), NC_002764 for Barbary macaque (Macaca sylvanus), NC_002763 for whitefronted capuchin (Cebus albifrons), NC_002765 for slow loris (Nycticebus coucang), NC_002521 for northern tree shrew (Tupaia belangeri), and NC_001325 for harbor seal (Phoca vitulina).

Branch lengths were calculated through maximum-likelihood methodology with the DNAML program in PHYLIP 3.573 (Felsenstein 1993). ClustalW was used to align each of the 82 numts to the 10 mitochondrial sequences. Each numt was added to each of the nine branches on the lineage leading to the human genome, and by using DNAML we computed the likelihood of each of the nine resulting trees.

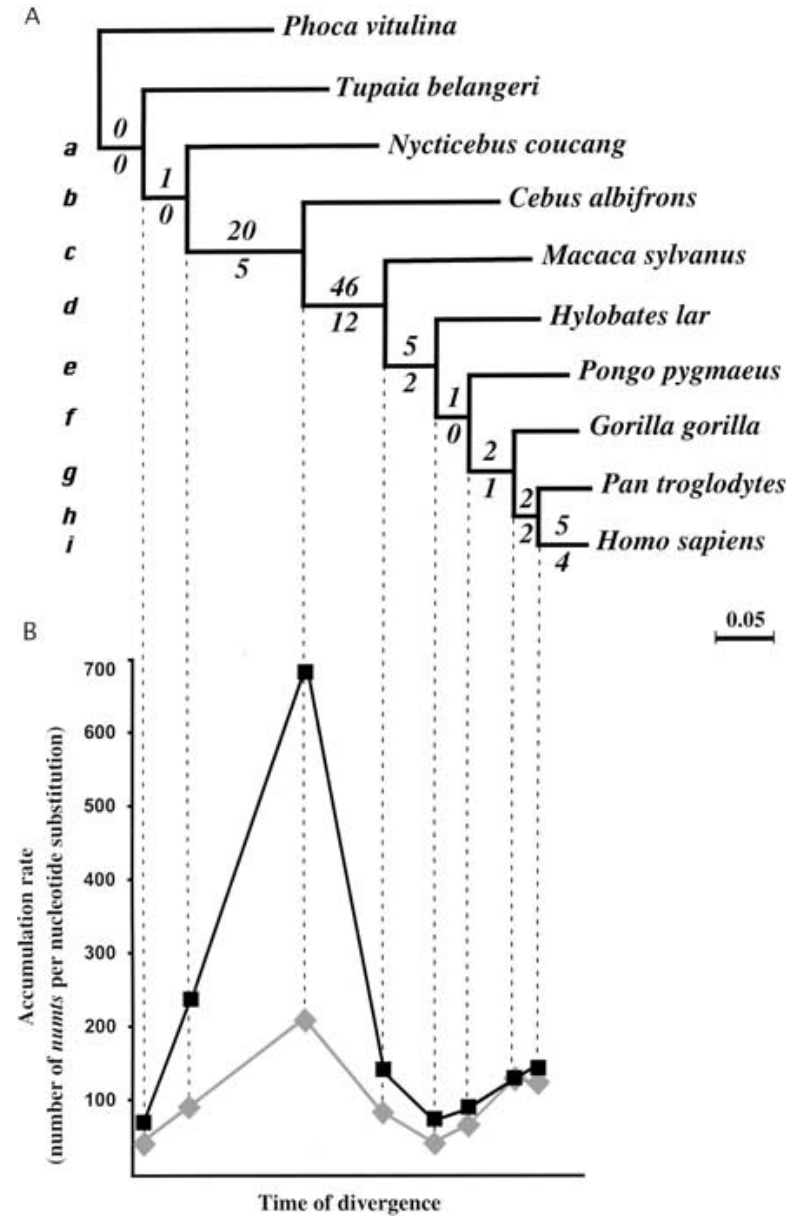

Fig. 1. (A) Maximum likelihood phylogenetic tree based on 10 complete mitochondrial sequences from primates and outgroups. Branch lengths were calculated with the DNAML program and are proportional to numbers of nucleotide substitutions in the mitochondria. Branch lengths are measured in units of nucleotide substitution per site (see bar). Numbers of numts that have originated at various evolutionary times (above branch), and number of separate insertions (below branch) are indicated. The notation for the tree branches $(a-i)$ is also used in Table 1. (B) Temporal dynamics of numt accumulation in the nuclear genome (black line), and those of separate numts insertions (gray line), plotted on a time axis derived from the maximum likelihood phylogenetic tree in $\mathbf{A}$. Time axis is measured in units of nucleotide substitution per site (see scale bar).

The nine trees were given two scores: (1) The unweighted score was the number of times that each of the nine trees emerged as the most likely tree. (2) The weighted score was calculated as follows: If the likelihood of the best tree was significantly different from the other trees, the tree was given a score of 1 . If two trees could not be shown to differ from each other in a statistically significant manner $(p<0.05)$, each of the two trees was given a score of 0.5 . If three trees could not be shown to differ from one another in a statistically significant manner, each of the three trees was given a score of 0.33 , and so on. For each of the nine trees, we summed the scores over the 82 numts.

Phylogenic Analysis of Pairs of numts. We compiled a database of pairs of numts, in which each pair contains a short numt that is fully contained within a long one. We used the previously determined maximum-likelihood branch location for the longer numt to identify the phylogenetic position of the shorter numt. The maximum likelihood position for the shorter numt in a pair was iden- 
tified with the user-tree option in the DNAML program. If the two numts emerged as sister taxa on the same branch, we concluded that the shorter numt represents a partial duplication of the longer one. In such a case, the longer numt is called the "father" and the shorter one is called the "son".

Inferrence of the Number of Independent numt Insertions. Numts that participate in pairs only as fathers but never as sons were deemed to have been created by insertion. Numts that did not appear in the database of pairs were also classified as independent insertions. All other numts were inferred to have been created by duplication of a preexisting numt.

\section{Results}

Eighty-two numts longer than 1,000 bp were identified in the human nuclear genome (Table 1). The chromosomal distribution of numts was found not deviate significantly from a random distribution $\left(\chi^{2}=22.85 ; d f=23 ; p \ll 0.47\right)$. This finding is in agreement with Mourier et al. (2001).

By using maximum likelihood methodology, it was possible to place each of the 82 numts in their temporal evolutionary context (Fig. 1A). When adjacent placements on the phylogenetic tree could not be distinguished from one another with sufficient statistical confidence, we assigned equal probability of numt origin on each of the indistinguishable branches. Numbers of numts were similar for both the weighted and the unweighted method.

We applied Grubbs' extreme studentized deviate test (Barnett and Lewis 1994) on the unweighted numbers of numts divided by the lengths of their respective branches. A statistically significant $(p<0.01) 30$-fold increase in the rate of numt accumulation was observed to have occurred on the branch leading to Catarrhini (Old World monkeys, apes, and humans) after their divergence from the Platyrrhini (New World monkeys) approximately 40 million years ago (Fig. 1B).

The dramatic change in the rate of numt accumulation could be due to increase in the rate of independent sequence transfers from the mitochondria to nucleus or due to post-insertion duplications within the nuclear genome. In order to distinguish between the two possibilities, we analyzed 323 numt pairs. Nine of the 82 numts were found to have no relation to the other numts and were, thus, considered as independent insertions. The other 73 numts were inferred to have been created by the duplication of 17 ancestral numts. Thus, only $30 \%$ of all numts in the human nuclear genome have been created by insertion; the others have accumulated by subsequent duplication.

We placed each of the 26 independently inserted numts on the branches of the mitochondrial phylogenetic tree (Fig. 1A). Again, we found a relative excess of numt accumulation (this time attributed solely to insertion) on the branch leading to Catarrhini after its divergence from the Platyrrhini (Fig.
1B). Nevertheless, Grubbs' extreme studentized deviate test is no longer statistically significant.

The ratios between the number of numts and the number of numt insertions on the branches ranged from 4 to 1, with the higher values obtained for the older branches. This indicates, that older numts have been duplicated more times than younger ones.

\section{Discussion}

Recently, several papers analyzing the full numt repertoire reported a continuous evolutionary transfer of mitochondrial sequences into the human nuclear genome. Mourier et al. (2001) used a combination of BLAST (Altschul et al. 1997) and DNA-block alignment (Jareborg et al. 1999), and found that the human nuclear genome contains 296 numts, 94 of which were longer than $1,000 \mathrm{bp}$. In our survey, we have only identified 82 such numts, most probably because of our more conservative criteria for inclusion. Although the method of Mourier et al. (2001) is suitable for the identification of the human numt repertoire, their phylogenetic analysis is, to say the least, inconclusive. First, Mourier et al. (2001) ignored the possibility of numt duplication. Second, since many of their numts consisted of disjointed segments, in many cases numts were placed in more than one phylogenetic position on the tree. This is evolutionarily impossible and should be regarded as an artifact of their use of the block-alignment algorithm, which has yielded numts with varying degrees of similarity to the mitochondrial parent.

In the study by Woischnik and Moraes (2002), the authors searched for hits of single mitochondrial genes in the nuclear genome, and used their coordinates to combine them into longer numts. Woischnik and Moraes (2002) discovered 612 numts. The phylogenetic analysis in Woischnik and Moraes (2002) was carried out gene by gene, so that parts of the same numt were most probably positioned on different branches of the tree. And again, the possibility of numt duplication occurring subsequent to the insertion of the mitochondrial sequence was ignored.

Here, we performed an analysis on 82 long numts. We did not aim to identify the entire numt repertoire, a process that was most probably completed by Mourier et al. (2001), but to reconstruct numt evolutionary history by taking into account the possibility of genomic duplication. We found that the number of numts is positively correlated with branch length. For example, the longest branches, i.e., those representing the divergence between Platyrrhini and Catarrhini and between Strepsirhini and the rest of the Primates, show the higher number of numts. In other words, our analysis indicates that numt insertion into the nuclear genome is a continuous and largely monotonic evolutionary process. However, our analysis also indi- 
Table 1. Human numts longer than $1,000 \mathrm{bp}$

\begin{tabular}{|c|c|c|c|c|c|c|}
\hline Contig & Chromosome & Length & Mitochondria position & Contig position & $\%$ Similarity & Tree location $^{\mathrm{a}}$ \\
\hline NT_023115.7 & 5 & 8821 & $6390-15211$ & $3721-12548$ & $88.80 \%$ & $\mathrm{~g}$ \\
\hline NT_007412.7 & 6 & 5888 & $3912-9800$ & $141462-135572$ & $98.18 \%$ & $\mathrm{i}$ \\
\hline NT_030001.2 & 7 & 5831 & $6149-11980$ & $1337640-1331818$ & $59.52 \%$ & $\mathrm{~d}$ \\
\hline NT_009184.7 & 11 & 5765 & $9821-15586$ & $121983-116221$ & $59.79 \%$ & $\mathrm{~d}$ \\
\hline NT_004836.7 & 1 & 5634 & $573-6207$ & $1451784-1457418$ & $75.15 \%$ & $\mathrm{~d}$ \\
\hline NT_010530.7 & 16 & 5302 & $8584-13886$ & $2334047-2328751$ & $70.91 \%$ & $\mathrm{~d}$ \\
\hline NT_007091.7 & 5 & 5222 & $10266-15488$ & $588077-582855$ & $93.99 \%$ & $\mathrm{i}$ \\
\hline NT_024089.7 & 10 & 5177 & $3294-8543$ & $678570-684680$ & $61.50 \%$ & $\mathrm{c}$ \\
\hline NT_022208.5 & 2 & 4654 & $11590-16244$ & $178103-182758$ & $71.30 \%$ & $\mathrm{~d}$ \\
\hline NT_028400.2 & $\mathrm{X}$ & 4177 & $2232-6409$ & $179660-183822$ & $74.01 \%$ & $\mathrm{~d}$ \\
\hline NT_023451.7 & 6 & 4027 & $7669-11696$ & $1133421-1137446$ & $54.89 \%$ & $\mathrm{c}$ \\
\hline NT_022140.7 & 2 & 3827 & $8296-12123$ & $91132-94949$ & $75.70 \%$ & $\mathrm{~d}$ \\
\hline NT_0066129.6 & 4 & 3712 & $8296-12008$ & $427566-423870$ & $74.47 \%$ & $\mathrm{~d}$ \\
\hline NT_024862.6 & 17 & 3697 & $2141-5838$ & $2611-6307$ & $84.66 \%$ & $\mathrm{e}$ \\
\hline NT_006654.7 & 5 & 3464 & $12661-16125$ & $1068972-1072433$ & $86.56 \%$ & $\mathrm{~g}$ \\
\hline NT_006129.6 & 4 & 3379 & $663-4042$ & $99606-96231$ & $79.44 \%$ & $\mathrm{~d}$ \\
\hline NT_030719.1 & 7 & 3356 & $3293-6649$ & $24338-27690$ & $63.92 \%$ & $\mathrm{~d}$ \\
\hline NT_026437.5 & 14 & 3305 & $12417-15412$ & $2347244-2343953$ & $66.51 \%$ & $\mathrm{~d}$ \\
\hline NT_005151.7 & 2 & 3291 & $11802-15093$ & $2651955-2648663$ & $68.82 \%$ & $\mathrm{~d}$ \\
\hline NT_007995.7 & 8 & 3086 & $2009-5095$ & 191614-188537 & $62.55 \%$ & $\mathrm{~d}$ \\
\hline NT_011362.7 & 20 & 2766 & $1045-3811$ & 20988535-20985785 & $70.75 \%$ & $\mathrm{~d}$ \\
\hline NT_005229.7 & 2 & 2751 & $10409-13160$ & $1250750-1248006$ & $75.73 \%$ & $\mathrm{~d}$ \\
\hline NT_005129.7 & 2 & 2603 & $737-3340$ & $2165807-2168408$ & $71.33 \%$ & $\mathrm{~d}$ \\
\hline NT_023678.6 & 8 & 2547 & $1033-3580$ & $412979-410448$ & $74.39 \%$ & $\mathrm{~d}$ \\
\hline NT_007769.5 & 7 & 2545 & $576-3121$ & $224815-222274$ & $83.35 \%$ & $\mathrm{~d}$ \\
\hline NT_030040.2 & 9 & 2545 & $576-3121$ & $2066695-2069239$ & $83.56 \%$ & $\mathrm{~d}$ \\
\hline NT_009243.7 & 11 & 2451 & $523-2974$ & $832183-829734$ & $94.09 \%$ & $\mathrm{~h}$ \\
\hline NT_0085541.7 & 9 & 2443 & $4548-6991$ & $595250-597696$ & $75.80 \%$ & $\mathrm{~d}$ \\
\hline NT_022852.7 & 4 & 2432 & $12890-15322$ & $852792-850357$ & $75.50 \%$ & $\mathrm{e}$ \\
\hline NT_007884.7 & 7 & 2392 & $13066-15458$ & $437318-434933$ & $73.82 \%$ & $\mathrm{~d}$ \\
\hline NT_022790.7 & 4 & 2366 & $577-2943$ & $689245-691612$ & $68.46 \%$ & $\mathrm{~d}$ \\
\hline NT_011896.7 & $\mathrm{Y}$ & 2333 & $14237-16570$ & $5697142-5699469$ & $61.46 \%$ & $\mathrm{c}$ \\
\hline NT_008583.7 & 10 & 2309 & $11645-13954$ & $3338266-3340570$ & $74.51 \%$ & $\mathrm{~d}$ \\
\hline NT_024814.5 & 16 & 2303 & $13905-16208$ & 204841-202534 & $71.53 \%$ & $\mathrm{c}$ \\
\hline NT_006961.7 & 5 & 2281 & $420-2701$ & $103574-101297$ & $93.59 \%$ & $\mathrm{~h}$ \\
\hline NT_008583.7 & 10 & 2173 & $1703-3876$ & $2182212-2184389$ & $77.92 \%$ & $\mathrm{~d}$ \\
\hline NT_008251.7 & 8 & 2107 & $13932-16039$ & $858254-856146$ & $76.97 \%$ & $\mathrm{e}$ \\
\hline NT_023290.4 & 5 & 2099 & $5891-7990$ & $178922-181031$ & $70.80 \%$ & $\mathrm{~d}$ \\
\hline NT_009952.7 & 13 & 2048 & $13942-15990$ & $205485-207526$ & $72.84 \%$ & $\mathrm{~d}$ \\
\hline NT_0065576.7 & 5 & 2034 & $14139-16173$ & $1204488-1206530$ & $71.44 \%$ & $\mathrm{c}$ \\
\hline NT_005229.7 & 2 & 1995 & $9473-11468$ & $1588832-1590824$ & $64.54 \%$ & $\mathrm{c}$ \\
\hline NT_011649.7 & $\mathrm{X}$ & 1993 & $14031-16024$ & $1271724-1269724$ & $74.75 \%$ & $\mathrm{~d}$ \\
\hline NT_019350.7 & 3 & 1984 & $8619-10603$ & $936218-934237$ & $73.07 \%$ & $\mathrm{c}$ \\
\hline NT_011613.7 & $\mathrm{X}$ & 1893 & $14678-16571$ & $56571-54679$ & $65.36 \%$ & $\mathrm{~d}$ \\
\hline NT_008150.6 & 8 & 1868 & $4865-6733$ & $812806-814667$ & $75.75 \%$ & $\mathrm{~d}$ \\
\hline NT_006322.7 & 4 & 1861 & $9441-11302$ & $515297-517150$ & $73.51 \%$ & $\mathrm{~d}$ \\
\hline NT_015360.7 & 16 & 1833 & $2783-4616$ & $18822-16998$ & $76.03 \%$ & $\mathrm{~d}$ \\
\hline NT_026437.5 & 14 & 1810 & $575-2385$ & 2931912-2933718 & $65.18 \%$ & $\mathrm{~d}$ \\
\hline NT_006936.7 & 5 & 1770 & $14274-16044$ & 198918-197149 & $69.68 \%$ & $\mathrm{c}$ \\
\hline NT_005638.6 & 3 & 1669 & $9105-10774$ & $622432-624101$ & $75.03 \%$ & $\mathrm{~d}$ \\
\hline NT_027070.4 & 8 & 1631 & $10772-12403$ & $775266-773640$ & $73.92 \%$ & $\mathrm{~d}$ \\
\hline NT_005112.7 & 2 & 1609 & $10995-12604$ & $158769-157171$ & $74.83 \%$ & $\mathrm{~d}$ \\
\hline NT_025395.6 & $\mathrm{X}$ & 1584 & $12995-14579$ & $136603-135032$ & $61.78 \%$ & $\mathrm{c}$ \\
\hline NT_008218.7 & 8 & 1553 & $7024-8577$ & $7163-8721$ & $94.23 \%$ & $\mathrm{i}$ \\
\hline NT_030590.1 & 2 & 1531 & $8272-9803$ & $339514-341038$ & $71.87 \%$ & $\mathrm{c}$ \\
\hline NT_009151.7 & 11 & 1470 & $8309-9779$ & $6648376-6646914$ & $73.00 \%$ & $\mathrm{c}$ \\
\hline NT_030828.1 & 15 & 1452 & $11683-13135$ & $3728186-3729632$ & $75.77 \%$ & $\mathrm{~d}$ \\
\hline NT_024862.6 & 17 & 1448 & $14335-15783$ & $139720-138272$ & $82.29 \%$ & $\mathrm{f}$ \\
\hline NT_010441.7 & 16 & 1446 & $12196-13642$ & $244421-242976$ & $74.90 \%$ & $\mathrm{~d}$ \\
\hline NT_030761.1 & 9 & 1417 & $8474-9891$ & $1556287-1554877$ & $74.44 \%$ & $\mathrm{~d}$ \\
\hline NT_022475.4 & 3 & 1384 & $1387-2771$ & $511841-510458$ & $93.07 \%$ & $\mathrm{i}$ \\
\hline NT_022066.1 & 1 & 1368 & $14671-16039$ & $76000-74633$ & $77.24 \%$ & $\mathrm{e}$ \\
\hline NT_005151.7 & 2 & 1353 & $4855-6208$ & $2698233-2699587$ & $81.59 \%$ & $\mathrm{e}$ \\
\hline NT_009184.7 & 11 & 1337 & $14505-15842$ & $6619287-6617962$ & $72.73 \%$ & $\mathrm{c}$ \\
\hline
\end{tabular}


Table I. Continued

\begin{tabular}{lllllll}
\hline Contig & Chromosome & Length & Mitochondria position & Contig position & \%Similarity $^{\text {Tree location }}{ }^{\mathrm{a}}$ \\
\hline NT_019350.7 & 3 & 1324 & $3501-4825$ & $939172-940487$ & $72.95 \%$ & $\mathrm{~d}$ \\
NT_030846.1 & 17 & 1306 & $3178-4484$ & $618593-617293$ & $76.95 \%$ & $\mathrm{~d}$ \\
NT_025766.6 & 7 & 1282 & $14748-16030$ & $707948-709233$ & $70.74 \%$ & $\mathrm{c}$ \\
NT_010289.7 & 15 & 1275 & $14465-15740$ & $2202527-2201254$ & $74.46 \%$ & $\mathrm{c}$ \\
NT_015326.7 & 12 & 1249 & $3923-5172$ & $860080-858838$ & $71.24 \%$ & $\mathrm{c}$ \\
NT_028068.4 & 2 & 1224 & $8398-9622$ & $460959-462176$ & $75.43 \%$ & $\mathrm{~d}$ \\
NT_026965.2 & 2 & 1221 & $13007-14228$ & $32515-33735$ & $72.42 \%$ & $\mathrm{~d}$ \\
NT_010530.7 & 16 & 1211 & $10474-11685$ & $2401941-2403161$ & $72.44 \%$ & $\mathrm{~d}$ \\
NT_005445.7 & 2 & 1153 & $15418-16571$ & $819365-818210$ & $63.75 \%$ & $\mathrm{c}$ \\
NT_004836.7 & 1 & 1110 & $13693-14803$ & $3956054-3957164$ & $70.59 \%$ & $\mathrm{c}$ \\
NT_024296.3 & 11 & 1084 & $6449-7533$ & $72596-73680$ & $75.16 \%$ & $\mathrm{~d}$ \\
NT_022497.7 & 3 & 1076 & $8275-9351$ & $541783-542854$ & $71.89 \%$ & $\mathrm{~d}$ \\
NT_011512.4 & 21 & 1050 & $4910-5960$ & $22840953-22839916$ & $64.87 \%$ & $\mathrm{c}$ \\
NT_011574.3 & $\mathrm{X}$ & 1040 & $753-1793$ & $969765-968737$ & $62.78 \%$ & $\mathrm{c}$ \\
NT_019284.6 & 1 & 1037 & $2071-3108$ & $225524-226553$ & $67.43 \%$ & $\mathrm{c}$ \\
NT_019350.7 & 3 & 1035 & $1015-2050$ & $941525-940486$ & $77.36 \%$ & $\mathrm{c}$ \\
NT_010164.7 & 14 & 1025 & $5584-6609$ & $6073254-6072232$ & $92.98 \%$ & \\
NT_006560.7 & 5 & 1008 & $6953-7961$ & $1103416-1104421$ & $78.12 \%$ & $\mathrm{~d}$ \\
\hline
\end{tabular}

${ }^{a}$ Tree location as in Fig. 1.

cates that the process of numt insertion is less frequent than previously reported. The peak in numt accumulation that is found on the branch representing the divergence of Platyrrhini from the Catarrhini is mostly the result of duplication of preexisting nuclear numts rather than the result of an increase in mitochondrial-sequence insertion. In fact, this phenomenon is a general one; on average only one of every three numts in our genome is the result of an independent integration event, while the other two originate from duplications within the nuclear genome.

Under the assumption that genomic duplications occur at rates that are uninfluenced by content, older numts should appear in larger copy numbers than recently inserted numts. Let us consider, for instance, branch $\mathrm{C}$ in Figure 1. Twenty numts were located on this branch, yet these 20 numts are derived from only five independent insertions. In contrast, of the five numts inferred to have accumulated in the human genome after the Homo-Pan divergence, four are most probably independent insertions. These five numts are expected to have no homologues in non-human genomes. The fact that older numts were indeed found to be duplicated more times than younger numts strengthens the confidence in the reliability of our results.

Acknowledgments. We wish to thank the people of Compugen Ltd., especially Idit Azar, Gad S. Cojocaru, and Erez Levanon. E. H.-C. wishes to thank Shay Covo for his helpful advise.

\section{References}

Altschul SF, Madden TL, Schaffer AA, Zhang J, Zhang Z, Miller W, Lipman DJ (1997) Gapped BLAST and PSI-BLAST: a new generation of protein database search programs. Nucleic Acids Res 25:3389-3402

Barnett V, Lewis T (1994) Outliers in statistical data. Wiley \&Sons, New York

Bensasson D, Zhang D, Hartl DL, Hewitt GM (2001) Mitochondrial pseudogenes: evolution's misplaced witnesses. Trends Ecol Evol 16:314-321

Bensasson D, Zhang DX, Hewitt GM (2000) Frequent assimilation of mitochondrial DNA by grasshopper nuclear genomes. Mol Biol Evol 17:406-415

Blanchard JL, Schmidt GW (1995) Pervasive migration of organellar DNA to the nucleus in plants. J Mol Evol 41:397-406

Boore JL (1999) Animal mitochondrial genomes. Nucleic Acids Res 27:1767-1780

Collura RV, Stewart CB (1995) Insertions and duplications of mtDNA in the nuclear genomes of Old World monkeys and hominoids. Nature 378:485-489

Ellis J (1982) Promiscuous DNA-chloroplast genes inside plant mitochondria. Nature 299:678-679

Felsenstein J (1993) PHYLIP: Phylogeny inference package and manual. Version 3.5 Department of Genetics, University of Washington, Seattle

Fukuda M, Wakasugi S, Tsuzuki T, Nomiyama H, Shimada K, Miyata T (1985) Mitochondrial DNA-like sequences in the human nuclear genome. Characterization and implications in the evolution of mitochondrial DNA. J Mol Biol 186: 257-266

Goodman M, Porter CA, Czelusniak J, Page SL, Schneider H, Shoshani J, et al. (1998) Toward a phylogenetic classification of Primates based on DNA evidence complemented by fossil evidence. Mol Phylogenet Evol 9:585-598

Henze K, Martin W (2001) How do mitochondrial genes get into the nucleus? Trends Genet 17:383-387

Higgins DG, Thompson JD, Gibson TJ (1996) Using CLUSTAL for multiple sequence alignments. Methods Enzymol 266:383402

Jareborg N, Birney E, Durbin R (1999) Comparative analysis of noncoding regions of 77 orthologous mouse and human gene pairs. Genome Res 9:815-824

Lewin R (1983) Promiscuous DNA leaps all barriers. Science 219:478-479 
Lopez JV, Yuhki N, Masuda R, Modi W, O’Brien SJ (1994) Numt, a recent transfer and tandem amplification of mitochondrial DNA to the nuclear genome of the domestic cat. J Mol Evol 39:174-190

Mourier T, Hansen AJ, Willerslev E, Arctander P (2001) The Human Genome Project reveals a continuous transfer of large mitochondrial fragments to the nucleus. Mol Biol Evol 18:1833-1837

Pearson WR, Lipman DJ (1988) Improved tools for biological sequence comparison. Proc Natl Acad Sci USA 85: 2444-2448
Smith TF, Waterman MS (1981) Identification of common molecular subsequences. J Mol Biol 147:195-197

Stern DB, Lonsdale DM (1982) Mitochondrial and chloroplast genomes of maize have a 12-kilobase DNA sequence in common. Nature 299:698-702

Thorsness PE, Weber ER (1996) Escape and migration of nucleic acids between chloroplasts, mitochondria, and the nucleus. Int Rev Cytol 165:207-234

Woischnik M, Moraes CT (2002) Pattern of organization of human mitochondrial pseudogenes in the nuclear genome. Genome Res 12:885-893 